\title{
Uniqueness Theorems on Entire Functions and Their Difference Operators or Shifts
}

\author{
Baoqin Chen, ${ }^{1}$ Zongxuan Chen, ${ }^{1}$ and Sheng $\mathrm{Li}^{2}$ \\ ${ }^{1}$ School of Mathematical Sciences, South China Normal University, Guangzhou 510631, China \\ ${ }^{2}$ College of Science, Guangdong Ocean University, Zhangjiang 524088, China
}

Correspondence should be addressed to Zongxuan Chen, chzx@vip.sina.com

Received 23 December 2011; Accepted 24 March 2012

Academic Editor: István Györi

Copyright (c) 2012 Baoqin Chen et al. This is an open access article distributed under the Creative Commons Attribution License, which permits unrestricted use, distribution, and reproduction in any medium, provided the original work is properly cited.

We study the uniqueness problems on entire functions and their difference operators or shifts. Our main result is a difference analogue of a result of Jank-Mues-Volkmann, which is concerned with the uniqueness of the entire function sharing one finite value with its derivatives. Two relative results are proved, and examples are provided for our results.

\section{Introduction and Main Results}

Throughout this paper, we assume the reader is familiar with the standard notations and fundamental results of Nevanlinna theory of meromorphic functions (see, e.g., [1-3]). In what follows, a meromorphic function always means meromorphic in the whole complex plane, and $c$ always means a nonzero complex constant. For a meromorphic function $f(z)$, we define its shift by $f(z+c)$ and its difference operators by

$$
\Delta_{c} f(z)=f(z+c)-f(z), \quad \Delta_{c}^{n} f(z)=\Delta_{c}^{n-1}\left(\Delta_{c} f(z)\right), \quad n \in \mathbb{N}, n \geq 2 .
$$

For a meromorphic function $f(z)$, we use $S(f)$ to denote the family of all meromorphic functions $a(z)$ that satisfy $T(r, a)=S(r, f)$, where $S(r, f)=o(T(r, f))$, as $r \rightarrow \infty$ outside of a possible exceptional set of finite logarithmic measure. Functions in the set $S(f)$ are called small functions with respect to $f(z)$.

Let $f(z)$ and $g(z)$ be two meromorphic functions, and let $a(z)$ be a small function with respect to $f(z)$ and $g(z)$. We say that $f(z)$ and $g(z)$ share $a(z)$ IM, provided that $f(z)-a(z)$ and $g(z)-a(z)$ have the same zeros (ignoring multiplicities), and we say that $f(z)$ and $g(z)$ share $a(z) \mathrm{CM}$, provided that $f(z)-a(z)$ and $g(z)-a(z)$ have the same zeros with the same multiplicities. 
Uniqueness theory of meromorphic functions is an important part of the Nevanlinna theory. In the past 40 years, a very active subject is the investigation on the uniqueness of the entire function sharing values with its derivatives, which was initiated by Rubel and Yang [4]. We first recall the following result by Jank et al. [5].

Theorem A (see [5]). Let $f$ be a nonconstant meromorphic function, and let $a \neq 0$ be a finite constant. If $f, f^{\prime}$, and $f^{\prime \prime}$ share the value a $C M$, then $f \equiv f^{\prime}$.

Recently, value distribution in difference analogues of meromorphic functions has become a subject of some interest (see, e.g., [6-11]). In particular, a few authors started to consider the uniqueness of meromorphic functions sharing small functions with their shifts or difference operators (see, e.g., [12, 13]).

In this paper, we consider difference analogues of Theorem A.

Theorem 1.1. Let $f(z)$ be a nonconstant entire function of finite order, and let $a(z)(\not \equiv 0) \in S(f)$ be a periodic entire function with period c. If $f(z), \Delta_{c} f$, and $\Delta_{c}^{2} f$ share $a(z) C M$, then $\Delta_{c}^{2} f=\Delta_{c} f$.

Example 1.2. Let $f(z)=e^{z \ln 2}$ and $c=1$. Then, for any $a \in \mathbb{C}$, we notice that $f(z), \Delta_{c} f$, and $\Delta_{c}^{2} f$ share $a \mathrm{CM}$ and can easily see that $\Delta_{c}^{2} f=\Delta_{c} f$. This example satisfies Theorem 1.1.

Remark 1.3. In Example 1.2, we have $\Delta_{c}^{2} f=\Delta_{c} f=f$. However, it remains open whether the claim $\Delta_{c}^{2} f=\Delta_{c} f$ in Theorem 1.1 can be replaced by $\Delta_{c} f=f$ in general. In fact, the next example resulted from our efforts to find an entire function $f(z)$ satisfying Theorem 1.1, while $\Delta_{c} f \neq f$.

Example 1.4. Let $f(z)=e^{z \ln 2}-2, a=-1, b=1$, and $c=1$. Then we observe that $f(z)-a=$ $e^{z \ln 2}-1, \Delta_{c} f-b=e^{z \ln 2}-1$, and $\Delta_{c}^{2} f-b=e^{z \ln 2}-1$ share 0 CM. Here, we also get $\Delta_{c}^{2} f=\Delta_{c} f$.

From this example, it is natural to ask what happens if $f(z)-a(z), \Delta_{c} f-b(z)$, and $\Delta_{c}^{2} f-b(z)$ share $0 \mathrm{CM}$, where $a(z)$ and $b(z)$ are two (not necessarily distinct) small periodic entire functions. Considering this question, we prove the following Theorem 1.5, whose proof is omitted as it is similar to the proof of Theorem 1.1.

Theorem 1.5. Let $f(z)$ be a nonconstant entire function of finite order, and let $a(z), b(z)(\not \equiv 0) \in$ $S(f)$ be periodic entire functions with period c. If $f(z)-a(z), \Delta_{c} f-b(z)$, and $\Delta_{c}^{2} f-b(z)$ share 0 $C M$, then $\Delta_{c}^{2} f=\Delta_{c} f$.

Now it would be interesting to know what happens if the difference operators of $f(z)$ are replaced by shifts of $f(z)$ in Theorem 1.5. We prove the following result concerning this question.

Theorem 1.6. Let $f(z)$ be a nonconstant entire function of finite order, let $a(z), b(z) \in S(f)$ be two distinct periodic entire functions with period $c$, and let $n$ and $m$ be positive integers satisfying $n>m$. If $f(z)-a(z), f(z+m c)-b(z)$, and $f(z+n c)-b(z)$ share $0 C M$, then $f(z+m c)=f(z+n c)$ for all $z \in \mathbb{C}$.

Example 1.7. Let $f(z)=\sin z+1, a=0, b=2$, and $c=\pi$. Then we notice that $f(z)-a=$ $\sin z+1, f(z+c)-b=-\sin z-1$, and $f(z+3 c)-b=-\sin z-1$ share 0 CM and can easily see that $f(z+c)=f(z+3 c)$ for all $z \in \mathbb{C}$. This example satisfies Theorem 1.6. 
Example 1.8. Let $f(z)=e^{z^{2}}+a(z)$, where $a(z) \in S(f)$ is a periodic entire function with period 1. Then $f(z)-a(z)=e^{z^{2}}, f(z+1)-a(z)=e^{(z+1)^{2}}$, and $f(z+3)-a(z)=e^{(z+3)^{2}}$ share $0 \mathrm{CM}$, while $f(z+c)-f(z+3 c) \not \equiv 0$. This example shows that the condition that $a(z)$ and $b(z)$ are distinct in Theorem 1.6 cannot be deleted.

\section{Proof of Theorem 1.1}

Lemma 2.1 (see [8, Theorem 2.1]). Let $f(z)$ be a meromorphic function of finite order $\rho$ and let $c$ be a nonzero complex constant. Then, for each $\varepsilon>0$,

$$
T(r, f(z+c))=T(r, f(z))+O\left(r^{\rho-1+\varepsilon}\right)+O(\log r) .
$$

Lemma 2.2 (see [10, Lemma 2.3]). Let $c \in \mathbb{C}, n \in \mathbb{N}$, and let $f(z)$ be a meromorphic function of finite order. Then for any small periodic function $a(z)$ with period $c$, with respect to $f(z)$,

$$
m\left(r, \frac{\Delta_{c}^{n} f}{f-a}\right)=S(r, f)
$$

where the exceptional set associated with $S(r, f)$ is of at most finite logarithmic measure.

Proof of Theorem 1.1. Suppose, on the contrary, the assertion that $\Delta_{c}^{2} f \neq \Delta_{c} f$. Note that $f(z)$ is a nonconstant entire function of finite order. By Lemma 2.1, $\Delta_{c} f$ and $\Delta_{c}^{2} f$ are entire functions of finite order.

Since $f(z), \Delta_{c} f$, and $\Delta_{c}^{2} f$ share $a(z) \mathrm{CM}$, then we have

$$
\frac{\Delta_{c}^{2} f-a(z)}{f(z)-a(z)}=e^{\alpha(z)}, \quad \frac{\Delta_{c} f-a(z)}{f(z)-a(z)}=e^{\beta(z)},
$$

where $\alpha(z)$ and $\beta(z)$ are polynomials.

Set

$$
\varphi(z)=\frac{\Delta_{c}^{2} f-\Delta_{c} f}{f(z)-a(z)}
$$

From (2.3), we get $\varphi(z)=e^{\alpha(z)}-e^{\beta(z)}$. Then by supposition and (2.4), we see that $\varphi(z) \not \equiv 0$. By Lemma 2.2, we deduce that

$$
\begin{aligned}
T(r, \varphi) & =m(r, \varphi) \\
& \leq m\left(r, \frac{\Delta_{c}^{2} f}{f(z)-a(z)}\right)+m\left(r, \frac{\Delta_{c} f}{f(z)-a(z)}\right)+\log 2=S(r, f) .
\end{aligned}
$$


Note that $e^{\alpha} / \varphi-e^{\beta} / \varphi=1$. By using the second main theorem and (2.5), we have

$$
\begin{aligned}
T\left(r, \frac{e^{\alpha}}{\varphi}\right) & \leq \bar{N}\left(r, \frac{e^{\alpha}}{\varphi}\right)+\bar{N}\left(r, \frac{\varphi}{e^{\alpha}}\right)+\bar{N}\left(r, \frac{1}{e^{\alpha} / \varphi-1}\right)+S\left(r, \frac{e^{\alpha}}{\varphi}\right) \\
& =\bar{N}\left(r, \frac{e^{\alpha}}{\varphi}\right)+\bar{N}\left(r, \frac{\varphi}{e^{\alpha}}\right)+\bar{N}\left(r, \frac{\varphi}{e^{\beta}}\right)+S\left(r, \frac{e^{\alpha}}{\varphi}\right) \\
& =S(r, f)+S\left(r, \frac{e^{\alpha}}{\varphi}\right) .
\end{aligned}
$$

Thus, by (2.5) and (2.6), we have $T\left(r, e^{\alpha}\right)=S(r, f)$. Similarly, $T\left(r, e^{\beta}\right)=S(r, f)$.

By Lemma 2.2 and the first equation in (2.3), we deduce that $a(z) /(f(z)-a(z))=$ $\Delta_{c}^{2} f /(f(z)-a(z))-e^{\alpha(z)}$ and

$$
\begin{aligned}
m\left(r, \frac{1}{f(z)-a(z)}\right) & =m\left(r, \frac{1}{a(z)}\left(\frac{\Delta_{c}^{2} f}{f(z)-a(z)}-e^{\alpha(z)}\right)\right) \\
& \leq m\left(r, \frac{\Delta_{c}^{2} f}{f(z)-a(z)}\right)+m\left(r, e^{\alpha(z)}\right)+S(r, f) \\
& =S(r, f) .
\end{aligned}
$$

From (2.7), we see that

$$
\begin{aligned}
N\left(r, \frac{1}{f(z)-a(z)}\right) & =T(r, f(z))-m\left(r, \frac{1}{f(z)-a(z)}\right)+S(r, f) \\
& =T(r, f(z))+S(r, f) .
\end{aligned}
$$

Now we rewrite the second equation in (2.3) as $\Delta_{c} f=e^{\beta(z)}(f(z)-a(z))+a(z)$ and deduce that

$$
\begin{aligned}
\Delta_{c}^{2} f & =\Delta_{c}\left(e^{\beta(z)}(f(z)-a(z))+a(z)\right) \\
& =e^{\beta(z+c)}(f(z+c)-a(z+c))+a(z+c)-e^{\beta(z)}(f(z)-a(z))-a(z) \\
& =e^{\beta(z+c)}(f(z+c)-a(z))-e^{\beta(z)}(f(z)-a(z)) .
\end{aligned}
$$

This together with the first equation in (2.3) gives

$$
\begin{aligned}
f(z+c)= & \left(e^{\alpha(z)-\beta(z+c)}+e^{\beta(z)-\beta(z+c)}\right) f(z) \\
& -a(z)\left(e^{\alpha(z)-\beta(z+c)}+e^{\beta(z)-\beta(z+c)}-1-e^{-\beta(z+c)}\right),
\end{aligned}
$$


that is,

$$
\begin{aligned}
\Delta_{c} f= & \left(e^{\alpha(z)-\beta(z+c)}+e^{\beta(z)-\beta(z+c)}-1\right) f(z) \\
& -a(z)\left(e^{\alpha(z)-\beta(z+c)}+e^{\beta(z)-\beta(z+c)}-1-e^{-\beta(z+c)}\right) .
\end{aligned}
$$

Thus, (2.11) can be rewritten as

$$
\Delta_{c} f=\gamma(z) f(z)+\delta(z)
$$

where

$$
\begin{aligned}
\gamma(z) & =e^{\alpha(z)-\beta(z+c)}+e^{\beta(z)-\beta(z+c)}-1, \\
\delta(z) & =-a(z)\left(e^{\alpha(z)-\beta(z+c)}+e^{\beta(z)-\beta(z+c)}-1-e^{-\beta(z+c)}\right) \\
& =-a(z) \gamma(z)+a(z) e^{-\beta(z+c)},
\end{aligned}
$$

which satisfy $T(r, \gamma)=S(r, f)$ and $T(r, \delta)=S(r, f)$.

Now we rewrite $\Delta_{c} f=\gamma(z) f(z)+\delta(z)$ as

$$
\Delta_{c} f-a(z)-\gamma(z)(f(z)-a(z))=\gamma(z) a(z)+\delta(z)-a(z) .
$$

Suppose that $\gamma(z) a(z)+\delta(z)-a(z) \not \equiv 0$. Let $z_{0}$ be a zero of $f(z)-a(z)$ with multiplicity $k$. Since $f(z), \Delta_{c} f$ share $a(z) \mathrm{CM}$, then $z_{0}$ is a zero of $\Delta_{c} f-a(z)$ with multiplicity $k$. Thus, $z_{0}$ is a zero of $\Delta_{c} f-a(z)-\gamma(z)(f(z)-a(z))$ with multiplicity at least $k$. Then, by (2.8) and (2.14), we see that

$$
\begin{aligned}
N\left(r, \frac{1}{r(z) a(z)+\delta(z)-a(z)}\right) & =N\left(r, \frac{1}{\Delta_{c} f-a(z)-r(z)(f(z)-a(z))}\right) \\
& \geq N\left(r, \frac{1}{f(z)-a(z)}\right) \\
& =T(r, f(z))+S(r, f) .
\end{aligned}
$$

On the other hand, we have

$$
N\left(r, \frac{1}{\gamma(z) a(z)+\delta(z)-a(z)}\right) \leq T\left(r, \frac{1}{\gamma(z) a(z)+\delta(z)-a(z)}\right)=S(r, f) .
$$

Then, by (2.15) and (2.16), we get $T(r, f) \leq S(r, f)$, which is a contradiction.

Thus, $\gamma(z) a(z)+\delta(z)-a(z) \equiv 0$. Noting that $\delta(z)=-a(z) \gamma(z)+a(z) e^{-\beta(z+c)}$, we deduce that $e^{-\beta(z+c)} \equiv 1$. So, $e^{\beta(z)} \equiv e^{\beta(z+c)} \equiv 1$, since $\beta(z)$ is a polynomial.

By the second equation in (2.3), we obtain $\Delta_{c} f=f$, which leads to $\Delta_{c}^{2} f=\Delta_{c} f$. This is a contradiction. The proof is thus completed. 


\section{Proof of Theorem 1.6}

Lemma 3.1 (see [9, Corollary 2.2]). Let $f(z)$ be a nonconstant meromorphic function of finite order, $c \in \mathbb{C}$ and $\delta<1$. Then

$$
m\left(r, \frac{f(z+c)}{f(z)}\right)=o\left(\frac{T(r+|c|, f)}{r^{\delta}}\right)
$$

for all $r$ outside of a possible exceptional set with finite logarithmic measure.

Proof of Theorem 1.6. Suppose, on the contrary, the assertion that $f(z+m c)-f(z+n c) \not \equiv 0$. Since $f(z)-a(z), f(z+m c)-b(z)$, and $f(z+n c)-b(z)$ share $0 \mathrm{CM}$, then we have

$$
\frac{f(z+n c)-b(z)}{f(z)-a(z)}=e^{\alpha(z)}, \quad \frac{f(z+m c)-b(z)}{f(z)-a(z)}=e^{\beta(z)}
$$

where $\alpha(z)$ and $\beta(z)$ are polynomials.

By (3.2), we obtain

$$
\frac{f(z+n c)-f(z+m c)}{f(z)-a(z)}=e^{\alpha(z)}-e^{\beta(z)} .
$$

Set $\psi(z)=\mathrm{e}^{\alpha(z)}-e^{\beta(z)}$. Then by supposition, we see that $\psi(z) \not \equiv 0$. By Lemma 3.1, we deduce that

$$
\begin{aligned}
T(r, \psi) & =m\left(r, \frac{f(z+n c)-f(z+m c)}{f(z)-a(z)}\right) \\
& \leq m\left(r, \frac{f(z+n c)-a(z+n c)}{f(z)-a(z)}\right)+m\left(r, \frac{f(z+m c)-a(z+m c)}{f(z)-a(z)}\right)+\log 2 \\
& =S(r, f) .
\end{aligned}
$$

Note that $e^{\alpha} / \psi-e^{\beta} / \psi=1$. Thus, using a similar method as in the proof of Theorem 1.1, we get $T\left(r, e^{\alpha}\right)=S(r, f)$ and $T\left(r, e^{\beta}\right)=S(r, f)$.

By Lemma 3.1 and the first equation in (3.2), we deduce that

$$
\begin{aligned}
m\left(r, \frac{1}{f(z)-a(z)}\right) & =m\left(r, \frac{1}{b(z)-a(z)}\left(\frac{f(z+n c)-a(z)}{f(z)-a(z)}-e^{\alpha(z)}\right)\right) \\
& \leq m\left(r, \frac{f(z+n c)-a(z+n c)}{f(z)-a(z)}\right)+m\left(r, e^{\alpha}\right)+S(r, f) \\
& =S(r, f) .
\end{aligned}
$$


From (3.5), we see that

$$
N\left(r, \frac{1}{f(z)-a(z)}\right)=T(r, f(z))+S(r, f)
$$

Now we rewrite the second equation in (3.2) as $f(z+m c)=e^{\beta(z)}(f(z)-a(z))+b(z)$ and deduce that

$$
\begin{aligned}
f(z+n c)= & e^{\beta(z+(n-m) c)}(f(z+(n-m) c)-a(z+(n-m) c)) \\
& +b(z+(n-m) c) .
\end{aligned}
$$

This together with the first equation in (3.2) gives

$$
\begin{aligned}
f(z+(n-m) c)= & e^{\alpha(z)-\beta(z+(n-m) c)} f(z) \\
& -a(z) e^{\alpha(z)-\beta(z+(n-m) c)}+a(z+(n-m) c),
\end{aligned}
$$

that is,

$$
\begin{aligned}
f(z+n c)= & e^{\alpha(z+m c)-\beta(z+n c)} f(z+m c) \\
& -a(z+m c) e^{\alpha(z+m c)-\beta(z+n c)}+a(z+n c) \\
= & e^{\alpha(z+m c)-\beta(z+n c)} f(z+m c)-a(z) e^{\alpha(z+m c)-\beta(z+n c)}+a(z) .
\end{aligned}
$$

Now we rewrite (3.9) as

$$
\begin{aligned}
f(z+n c)-b(z)-e^{\alpha(z+m c)-\beta(z+n c)}(f(z+m c)-b(z)) \\
=(b(z)-a(z))\left(e^{\alpha(z+m c)-\beta(z+n c)}-1\right) .
\end{aligned}
$$

Suppose that $e^{\alpha(z+m c)-\beta(z+n c)} \equiv 1$; then, by (3.9), we get $f(z+n c)=f(z+m c)$, which is a contradiction.

Now we have $e^{\alpha(z+m c)-\beta(z+n c)}-1 \not \equiv 0$. Then using a similar method as in the proof of Theorem 1.1, we can also get a contradiction and obtain that $f(z+m c)=f(z+n c)$ for all $z \in \mathbb{C}$. Thus, Theorem 1.6 is proved.

\section{Acknowledgments}

This work was supported by the National Natural Science Foundation of China (no. 11171119). The authors would like to thank the referee for valuable suggestions.

\section{References}

[1] W. K. Hayman, Meromorphic Functions, Oxford Mathematical Monographs, Clarendon Press, Oxford, UK, 1964. 
[2] I. Laine, Nevanlinna Theory and Complex Differential Equations, vol. 15 of de Gruyter Studies in Mathematics, Walter de Gruyter, Berlin, Germany, 1993.

[3] C.-C. Yang and H.-X. Yi, Uniqueness Theory of Meromorphic Functions, vol. 557 of Mathematics and its Applications, Kluwer Academic Publishers, Dordrecht, The Netherlands, 2003.

[4] L. A. Rubel and C.-C. Yang, "Values shared by an entire function and its derivative," in Complex Analysis, vol. 599 of Lecture Notes in Mathematics, pp. 101-103, Springer, Berlin, Germany, 1977.

[5] G. Jank, E. Mues, and L. Volkmann, "Meromorphe Funktionen, die mit ihrer ersten und zweiten Ableitung einen endlichen Wert teilen," Complex Variables, Theory and Application, vol. 6, no. 1, pp. 51-71, 1986.

[6] W. Bergweiler and J. K. Langley, "Zeros of differences of meromorphic functions," Mathematical Proceedings of the Cambridge Philosophical Society, vol. 142, no. 1, pp. 133-147, 2007.

[7] Z.-X. Chen, "Value distribution of products of meromorphic functions and their differences," Taiwanese Journal of Mathematics, vol. 15, no. 4, pp. 1411-1421, 2011.

[8] Y.-M. Chiang and S.-J. Feng, "On the Nevanlinna characteristic of $f(z+\eta)$ and difference equations in the complex plane," Ramanujan Journal, vol. 16, no. 1, pp. 105-129, 2008.

[9] R. G. Halburd and R. J. Korhonen, "Difference analogue of the lemma on the logarithmic derivative with applications to difference equations," Journal of Mathematical Analysis and Applications, vol. 314, no. 2, pp. 477-487, 2006.

[10] R. G. Halburd and R. J. Korhonen, "Nevanlinna theory for the difference operator," Annales Academix Scientiarium Fennicæ. Mathematica, vol. 31, no. 2, pp. 463-478, 2006.

[11] J.-L. Zhang, "Value distribution and shared sets of differences of meromorphic functions," Journal of Mathematical Analysis and Applications, vol. 367, no. 2, pp. 401-408, 2010.

[12] J. Heittokangas, R. Korhonen, I. Laine, J. Rieppo, and J. Zhang, "Value sharing results for shifts of meromorphic functions, and sufficient conditions for periodicity," Journal of Mathematical Analysis and Applications, vol. 355, no. 1, pp. 352-363, 2009.

[13] S. Li and Z.-S. Gao, "Entire functions sharing one or two finite values CM with their shifts or difference operators," Archiv der Mathematik, vol. 97, no. 5, pp. 475-483, 2011. 


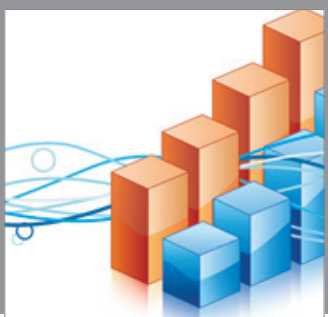

Advances in

Operations Research

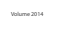

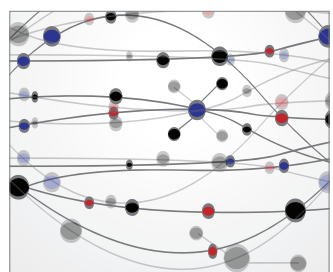

\section{The Scientific} World Journal
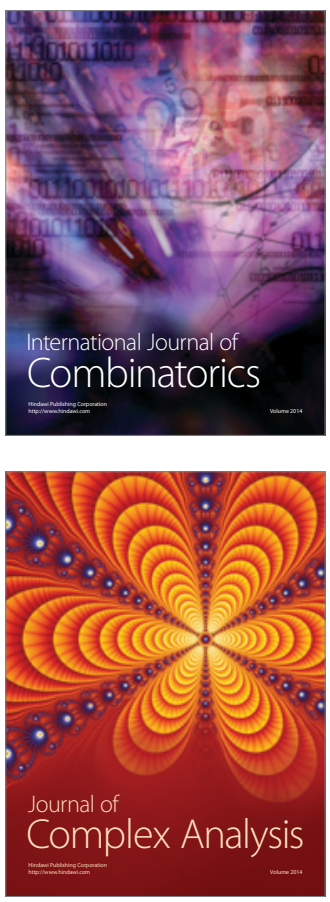

International Journal of

Mathematics and

Mathematical

Sciences
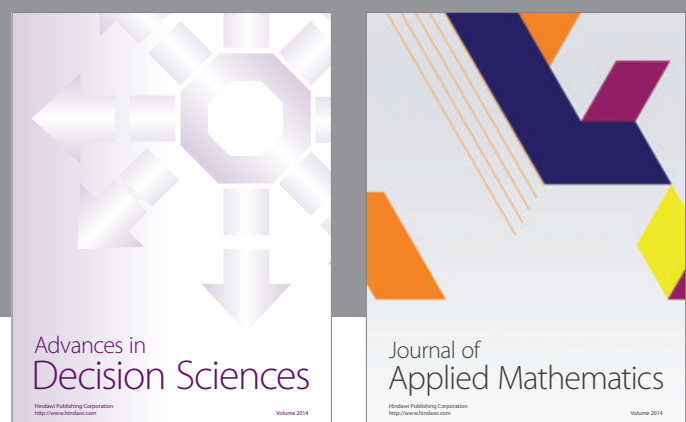

Journal of

Applied Mathematics
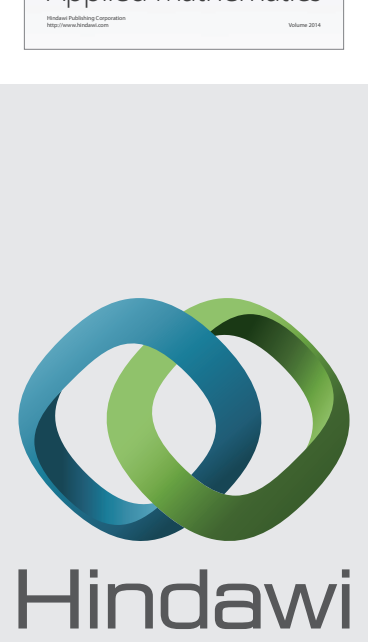

Submit your manuscripts at http://www.hindawi.com
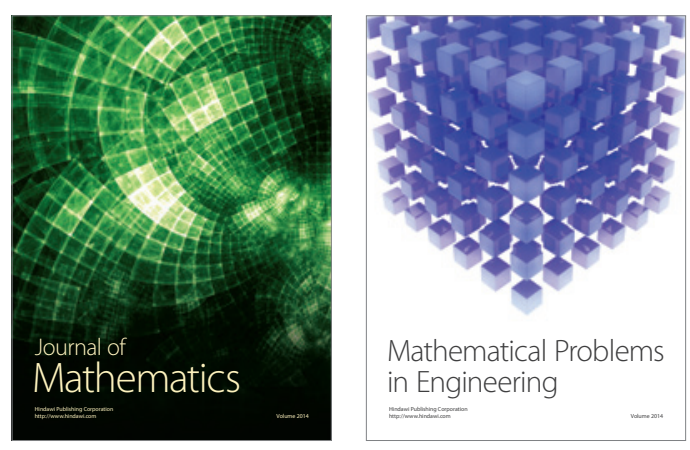

Mathematical Problems in Engineering
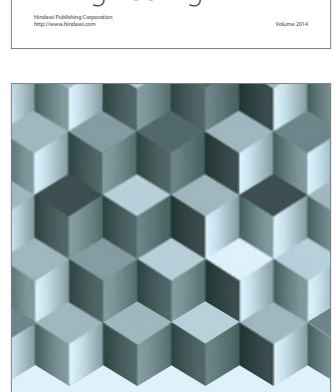

Journal of

Function Spaces
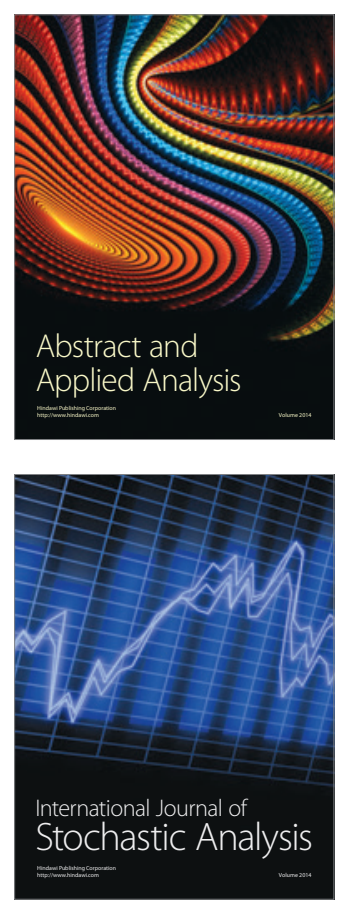

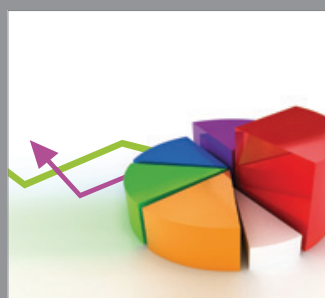

ournal of

Probability and Statistics

Promensencen
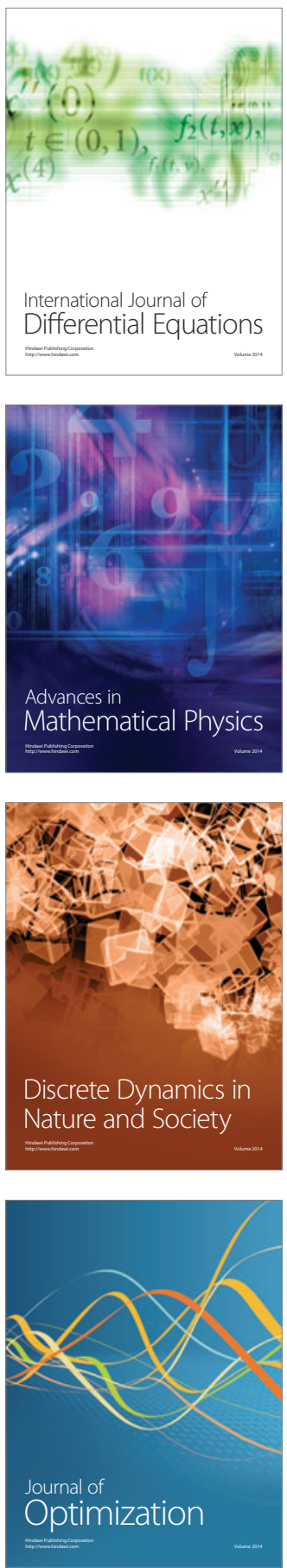